\section{Eating disorders}

\section{EPP0599}

\section{"Prevalence of orthorexia nervosa in a sample of patients attending sligo/leitrim mental health services with a diagnosis of eating disorder"}

\author{
I. Graffeo ${ }^{1 \star}$, M. Harron ${ }^{2}$ and E. O’Mahony ${ }^{2}$ \\ ${ }^{1}$ Sligo/leitrim Mental Health Services, St Columba's Hospital, Sligo, \\ Ireland and ${ }^{2}$ Psychiatry, Sligo/Leitrim Mental Health Services, Sligo, \\ Ireland \\ ${ }^{\star}$ Corresponding author. \\ doi: 10.1192/j.eurpsy.2021.940
}

Introduction: The term Orthorexia derives from the Greek "ortho - correct" and "orexis - appetite"; Orthorexia Nervosa is a pathological fixation with healthy eating that, starting with the idea to obtain a maximum health with a proper diet, leads to malnourishment and other medical sequelae, loss of relationships, loss of selfesteem, poor quality of life in general. Orthorexia, despite receiving broad empirical evidence, is not currently included in any psychiatric diagnostic manual.

Objectives: The main aim of this study is to investigate its presence in a sample of patients already diagnosed with a canonical eating disorder and also to understand eventual overlaps with other clinical disorders in order to optimize treatment and follow up.

Methods: The ORTO-15 questionnaire, developed by an Italian team of researchers in 2005, was used to achieve the above aims: it is a tool comprehensive of 15 questions that assesses eating habits perceived as healthy. Really interesting and fascinating is to comprehend if people with a diagnosis of eating disorder present orthorectic behaviour and how this emerging reality fits in the Irish society with its peculiarities and uniqueness.

Results: The Point Prevalence obtained is $17.9 \%$. The expected rates of Orthorexia Nervosa in the general population are between $6.9 \%$ and $57.6 \%$, with a peak of $81.8 \%$ in specific populations, fact that places our examined sample in the lower side of the prevalence previously considered in other studies.

Conclusions: It is significant the absence of correlation found between OCD and ON and that ON is more linked to Bulimia Nervosa rather than Anorexia Nervosa.

Keywords: eating disorders; orthorexia nervosa; anorexia nervosa; ocd

\section{EPP0597}

\section{Evidence-based therapeutic management of binge-eating disorder}

D. Vasile* and O. Vasiliu

Psychiatry, University Emergency Central Military Hospital Dr. Carol Davila, Bucharest, Romania

${ }^{\star}$ Corresponding author.

doi: 10.1192/j.eurpsy.2021.941

Introduction: Binge-eating disorder (BED) is a difficult-to-manage clinical entity, that may associate both organic (e.g., obesity, metabolic syndrome) and psychiatric (e.g., anxiety, mood, or substance use disorders) co-morbidity. Psychotherapeutic and pharmacological approaches are usually combined in order to reach the best outcome for these patients, but the disorder seems to have prolonged evolution even under appropriate therapeutic managment.

Objectives: To evaluate the most evidence-based therapies focused on BED.

Methods: A literature review was conducted through main electronic databases, and papers published between January 2000 and August 2020 were included in the analysis.

Results: Cognitive-behavioral therapy (CBT) is supported by multiple trials, and it led to decreased number of binge episodes and increased rates of remission. Behavioral activation may improve certain symptoms of BED (depressive mood, anxiety), but not the binge episodes frequency. Interpersonal group therapy (IPT) may be helpful for BED patients with an external locus of control and significant interpersonal dificulties. Dialectical behavior therapy (DBT) has been applied in BED patients, but the results have been inconclusive. Lisdexamfetamine dimesylate is the only FDA approved drug for this indication, as dasotraline was rejected by FDA and its research discontinued by the manufacturer. Fluoxetine, sertraline, escitalopram, duloxetine, bupropion, atomoxetine, reboxetine, armodafinil, disulfiram, baclofen, zonisamide, lamotrigine, topiramate, samidorphan, liraglutid, and orlistat need more trials in order to validate their efficacy, especially on long term.

Conclusions: There is only one drug currently FDA approved for this indication, lisdexamfetamine, and a number of psychotherapies, with CBT and IPT being the most supported by evidence.

Keywords: binge-eating disorder; psychotherapy;

lisdexamfetamine; antidepressants

\section{EPP0599}

Comparison of neuropsychological profiles in children and adolescent with anorexia nervosa and avoidant/ restrictive food intake disorder (ARFID)

C. Basile ${ }^{1 \star}$, F. Gigliotti $^{1}$, M. Colaiori ${ }^{2}$, F. Di Santo ${ }^{2}$, A. Terrinoni ${ }^{3}$, I. Ardizzone ${ }^{4}$ and U. Sabatello ${ }^{3}$

${ }^{1}$ Department Of Human Neuroscience,section Of Child And Adolescent Neuropsychiatry, Sapienza University of Rome, Roma, Italy; ${ }^{2}$ Department Of Human Neuroscience, Section Of Child And Adolescent Neuropsychiatry, Sapienza University of Rome, Roma, Italy; ${ }^{3}$ Department Of Human Neuroscience, Sapienza University of Rome, Section of Child and Adolescent Neuropsychiatry, RM, Italy and ${ }^{4}$ Department Of Human Neuroscience, Sapienza University of Rome, Roma, Italy

${ }^{*}$ Corresponding author.

doi: 10.1192/j.eurpsy.2021.942

Introduction: Anorexia Nervosa (AN) is an eating disorder characterized by low body weight, fear of gaining weight and distorted perception of body. Patients have rigidity, repetition of thoughts, alterations in decision-making skills and poor ability to provide new solutions. Avoidant/Restrictive Food Intake Disorder (ARFID) is a new eating disorder characterized by the absence of distress about body shape or fear of weight gain. Studies on neurocognitive aspects are few and no effective treatments are known.

Objectives: The aim of our study was to further investigate the executive functions' domains in AN and ARFID children and adolescents, to provide possible distinct neurocognitive traits in these patients. 
Methods: AN or ARFID patients ( $15+15$; range 6-18 years), were assessed by neuropsychological tools, such as: Wechsler Intelligence Scale to measure I.Q. profile, NEPSY-II to explore attention and executive functions, Tower of London test to detect planning and problem solving abilities, the Bells Test to evaluate visual selective and focused attention, the Wisconsing Card Sorting Test (WCST) for assessment of flexibility and directing behaviors by achieving a goal and the Rey-Osterrieth complex figure test (ROCF) to assess visual-spatial abilities.

Results: Patients with ARFID presented impairments in several executive functions domains, with difficulties in the impulse inhibition, in the sustained attention and in visual-spatial skills. Finally, in their anamnesis a higher comorbidity with neurodevelopmental disorders such as specific learning disorder has been underlined.

Conclusions: The identification of specific deficit in neuropsychological profile of ARFID patients could be a rehabilitation target, together with standardized treatment.

Keywords: Avoidant/Restrictive Food Intake Disorder; anorexia nervosa; neurodevelopment; executive function

\section{EPP0601}

\section{Microbiome-metabolomics signature in anorexia nervosa (AN) before and after weight regain}

F. Marciello ${ }^{1 \star}$, A.M. Monteleone ${ }^{2}$, J. Troisi ${ }^{3}$, G. Cascino ${ }^{1}$, G. Serena ${ }^{4}$, R. Dalle Grave ${ }^{5}$ and P. Monteleone ${ }^{6}$

${ }^{1}$ Department Of Medicine, Surgery And Dentistry, University of Salerno, Baronissi/Salerno, Italy; ${ }^{2}$ Department Of Psychiatry, University of Campania "Luigi Vanvitelli", Naples, Italy; ${ }^{3}$ (ebris), European Biomedical Research Institute of Salerno, Salerno, Italy; ${ }^{4}$ Mucosal Immunology And Biology Research Center, Massachusetts General Hospital-Harvard Medical School, Boston, United States of America; ${ }^{5}$ Department Of Eating And Weight Disorders, Villa Garda Hospital, Garda/Verona, Italy and ${ }^{6}$ Department Of Medicine, Surgery And Dentistry, University of Salerno, Baronissi/Salerno, Italy

${ }^{*}$ Corresponding author.

doi: 10.1192/j.eurpsy.2021.943

Introduction: The intestinal microbiota has been indicated to have a role in the pathophysiology of AN.

Objectives: Aim of this study was to analyze fecal microbiome profiles of $\mathrm{AN}$ women before and after weight restoration and to combine them with fecal metabolomic profiles according to a multi-omics approach.

Methods: The gut microbiome and fecal metabolites were characterized in 21 underweight $\mathrm{AN}$ women and after weight restoration and compared with those of 20 healthy women. Microbiome data were correlated with the relevant fecal metabolites.

Results: AN subjects showed a decreased intra-individual bacterial richness, an increased Bacteroidetes-to-Firmicutes abundance ratio and significant changes in the relative abundances of several bacteria at different order levels in both the underweight and weight-restored condition compared to healthy women. The untargeted metabolomic procedure allowed the characterization of 224 metabolites involved in energy, lipid and amino acid metabolism. A genetic algorithm identified 49 relevant metabolites. The relationships among these fecal metabolites and bacteria genera showed structures of different complexity in the 3 groups. In particular, a quarter of those relationships showed a divergent direction in the acute phase of $\mathrm{AN}$ than in the weight-restored phase or normal controls. Finally, in acute AN $70 \%$ of those correlations showed a negative sign suggesting a prevalent metabolites consummation by gut microbiome.

Conclusions: Our results provide a picture of the connections between gut bacteria and fecal metabolites in both the acute phase of AN and after short-term weight restoration. Further studies should aim to investigate the significance of gut microbiome perturbations in development and treatment of AN.

Keywords: anorexia nervosa; Gut Microbiota; eating disorders; Metabolomic

\section{EPP0602}

Equestrian vaulting as an innovative complementary intervention in eating disorders: A pilot study

B. Collacchi ${ }^{1}$, F. Cirulli ${ }^{1}$, M. Borgi ${ }^{1}$, I. Monaci ${ }^{2}$, A. Piccotti ${ }^{2}$, S. Renga ${ }^{3}$, L. Dalla Ragione ${ }^{4}$, M. Ettorre ${ }^{4}$, G. Biccheri ${ }^{4}$, F. Rossetti ${ }^{4}$ and S. Cerino ${ }^{5 *}$

${ }^{1}$ Center For Behavioural Sciences And Mental Health, Istituto Superiore di Sanità, er for Behavioural Sciences and Mental Health, Rome, Italy; ${ }^{2}$ Department Of Medicine, University of Perugia, Perugia, Italy; ${ }^{3}$ Psychology, Family Psychotherapy Academy, Rome, Italy; ${ }^{4}$ Umbertide Eating Disorder, USL Umbria 1, Perugia, Italy and ${ }^{5}$ Sphere, ECOS - EU, Massa Martana, Italy

${ }^{*}$ Corresponding author.

doi: 10.1192/j.eurpsy.2021.944

Introduction: Anorexia is a disorder associated with severe disturbances in eating behaviors and related thoughts and emotions (distorted weight perception, body dissatisfaction). Multidimensional integrative treatment approaches are needed to act both on intrapersonal (e.g. nutritional and psychological) and interpersonal (e.g. behavioral and affective) processes.

Objectives: Aim of this pilot project was to develop a 3-months horse-assisted intervention based on Equestrian Vaulting (EV) and tests its suitability and acceptability in patients with anorexia nervosa. Preliminary observations were carried out to assess the effectiveness of this program on body image, interpersonal relationships and communication and in managing anxiety.

Methods: Seven patients in charge of public service specialized in eating disorder participated in the study. EV activities were performed in an Equestrian Centre included horse grooming, gym exercises and horseback sessions.Clinical and psychological tests (SF 36, IPAQ, EDI3, STAI, SCL90) were administered at baseline and at the end of the program.

Results: Increases in body fat and decreases in lean muscle mass were observed. These were accompained by an improvement in participants' anxiety and relational skills and in the specific disease related symptoms.

Conclusions: Results indicate the potential of EV to help patients with eating disorder regaining awareness of themselves and their body, a critical element for their future reintegration in the contexts of everyday life and society. Although this is a pilot, the protocol developed represents an initial step to promote the application of EV in persons with eating disorders, informing feasibility in the design of larger controlled studies and suggesting critical variables to be targeted.

Keywords: eating disorder; psychiatric rehabilitation; equestrian sport 